\title{
Numerical Quadrature Rules for Some Infinite Range Integrals
}

\author{
By Avram Sidi
}

\begin{abstract}
Recently the present author has given a new approach to numerical quadrature and derived new numerical quadrature formulas for finite range integrals with algebraic and/or logarithmic endpoint singularities. In the present work this approach is used to derive new numerical quadrature formulas for integrals of the form $\int_{0}^{\infty} x^{\alpha} e^{-x} f(x) d x$ and $\int_{0}^{\infty} x^{\alpha} E_{p}(x) f(x) d x$, where $E_{p}(x)$ is the exponential integral. It turns out the new rules are of interpolatory type, their abscissas are distinct and lie in the interval of integration and their weights, at least numerically, are positive. For fixed $\alpha$ the new integration rules have the same set of abscissas for all $p$. Finally, the new rules seem to be at least as efficient as the corresponding Gaussian quadrature formulas. As an extension of the above, numerical quadrature formulas for integrals of the form $\int_{-\infty}^{+\infty}|x|^{\beta} e^{-x^{2}} f(x) d x$ too are considered.
\end{abstract}

1. Introduction. In a recent paper, [8], the present author has presented a new approach to numerical quadrature for finite range integrals with algebraic and/or logarithmic endpoint singularities. In particular, the integrals dealt with are of the form

$$
I[f]=\int_{0}^{1} w(x) f(x) d x,
$$

where $w(x)=(1-x)^{\alpha} x^{\beta}(-\log x)^{\nu}, \alpha+\nu>-1, \quad \beta>-1$, and the numerical quadrature formulas are of the form

$$
I_{k}[f]=\sum_{i=1}^{k} A_{k, i} f\left(x_{k, i}\right), \quad k=1,2, \ldots
$$

These formulas are based on some rational approximations obtained by applying a modification of the nonlinear sequence transformations due to Levin [6], to the moment series of $w(x)$ and seem to have some very important advantages which we now summarize.

(1) The abscissas $x_{k, i}$ are all in the interval $[0,1]$ and their weights turn out to be positive. For the weight functions $w(x)$ with $\alpha+\nu=0,1,2, \ldots$, that the abscissas are in $[0,1]$ has been proved.

(2) The abscissas are the same for a large class of endpoint singularities; in particular, they are independent of $\beta$ and depend solely on $\alpha+\nu$. Also if $\alpha+\nu$ is a small nonnegative integer like $0,1,2$, the abscissas $x_{k, i}$ for the case $w(x)=1$ can be taken to be the same for all these cases. In view of these remarks the weight functions $w(x)=1, w(x)=x^{\beta}, w(x)=x^{\beta}(-\log x), w(x)=x^{\beta}(-\log x)^{2}, w(x)=$ $x^{\beta}(1-x)^{1 / 2}(-\log x)^{-1 / 2}, w(x)=x^{\beta}(1-x)^{1 / 2}(-\log x)^{1 / 2}$, etc., all have the same set of abscissas regardless of what $\beta>-1$ is.

Received August 12, 1980; revised April 8, 1981.

1980 Mathematics Subject Classification. Primary 65D30, 65B05, 65B10, 40A05, 41A20. 
(3) The polynomials whose zeros are the abscissas $x_{k, i}$, are readily available in analytic form and the weights $A_{k, i}$ can be computed very easily once the abscissas have been determined.

(4) The new quadrature formulas have very strong convergence properties and are practically as efficient as the corresponding Gaussian formulas. For more details and numerical results the reader is referred to the paper of Sidi [8].

It turns out that the fruitful approach that has been used for the finite range integrals can be used for some infinite range integrals, among them some integrals that come up in radiation theory and involve the exponential integral as a weight function.

We shall now motivate the derivation of the new quadrature formulas for infinite range integrals in a manner analogous to that given in Sidi [8] and also exploit the motivation for introducing some of the notation to be used in the remainder of this work.

Let $f(z)$ be an analytic function in every finite subset of a simply connected open set $G$ of the complex plane which contains the real infinite interval $[0, \infty)$. Let also $\Gamma$ be a Jordan curve in $G$, containing the interval $[0, \infty)$ in its interior. Assume that $f(z)=O\left(z^{-a}\right)$ as $z \rightarrow \infty, z \in G$, for some $a>0$. Hence, by Cauchy's theorem, we have

$$
f\left(z_{0}\right)=\frac{1}{2 \pi i} \int_{\Gamma} \frac{f(z)}{z-z_{0}} d z,
$$

whenever $z_{0}$ is in the interior of $\Gamma$ and the integral is taken around $\Gamma$ in the counterclockwise direction.

Define the infinite range integral $J[f]$ and its $k$-point numerical quadrature rule $J_{k}[f]$ by

$$
J[f]=\int_{0}^{\infty} w(x) f(x) d x, \quad J_{k}[f]=\sum_{i=1}^{k} A_{k, i} f\left(x_{k, i}\right),
$$

where the abscissas $x_{k, i}$ are distinct. Define also

$$
H(z)=\int_{0}^{\infty} \frac{w(x)}{z-x} d x, \quad H_{k}(z)=\sum_{i=1}^{k} \frac{A_{k, i}}{z-x_{k, i}} .
$$

The function $H(z)$ is analytic in the $z$-plane cut along the positive real axis $[0, \infty)$ and has the divergent asymptotic expansion

$$
H(z) \sim \sum_{n=1}^{\infty} \frac{\mu_{n}}{z^{n}} \quad \text { as } z \rightarrow \infty, z \notin[0, \infty),
$$

where $\mu_{n}$ are the moments of $w(x)$,

$$
\mu_{n}=\int_{0}^{\infty} w(x) x^{n-1} d x, \quad n=1,2, \ldots
$$

Let $E_{k}[f]=J[f]-J_{k}[f]$. Then with the help of (1.3), (1.4), and (1.5) it can be shown that, when all the $x_{k, i}$ are in the interior of $\Gamma$,

$$
\left|E_{k}[f]\right| \leqslant \frac{1}{2 \pi} \int_{\Gamma}\left|H(z)-H_{k}(z)\right||f(z)||d z|,
$$

provided the integral on the right-hand side exists. Now if this integral tends to zero as $k \rightarrow \infty$, then $E_{k}[f] \rightarrow 0$ as $k \rightarrow \infty$. As mentioned in [8], in order for the 
integral in (1.8) and consequently for $E_{k}[f]$ to go to zero quickly as $k \rightarrow \infty$, we should demand that the sequence of the rational functions $H_{k}(z)$ tend to $H(z)$ uniformly and quickly in any region of the complex $z$-plane that is at a finite positive distance from the positive real axis.

If $H_{k}(z)$ is taken to be the $(k-1 / k)$ Padé approximant to (1.6), then $J_{k}[f]$ turns out to be the $k$-point Gaussian quadrature formula for $J[f]$. Furthermore, if $w(x) \geqslant 0$ on $[0, \infty)$, and the moments $\mu_{n}$ satisfy Carleman's condition, see Baker [2, Chapter 16] (this turns out to be the case for the $w(x)$ considered in this work), then as $k \rightarrow \infty, H_{k}(z) \rightarrow H(z)$ uniformly, in the sense of the previous paragraph.

In the present work we shall use again a modified version of the $T$-transformation of Levin to obtain another sequence of rational approximations $H_{k}(z)$, $k=1,2,3, \ldots$, to $H(z)$ from the series (1.6) and use these $H_{k}(z)$ to derive new numerical integration rules for some useful weight functions like $w(x)=x^{\alpha} e^{-x}$, $\alpha>-1$, and $w(x)=x^{\alpha} E_{p}(x), \alpha>-1, p+\alpha>0$, where $w(x)=E_{1}(x)$ and $w(x)=$ $E_{2}(x)$ come up in radiation theory; see Chandrasekhar [3, Chapter 2]. We shall also show that these new rules have advantages similar to those for the singular finite range integrals of [8], which we have summarized at the beginning of this section. We note that these advantages are a direct outcome of the results of a recent work by the author, [10].

The Gaussian quadrature formulas for the weight function $w(x)=x^{\alpha} e^{-x}, \alpha>$ -1 , have been widely tabulated. Gautschi [5] and Danloy [4] have considered the Gaussian quadrature rules for $w(x)=E_{p}(x), p>0$, and have given some tables for the case $p=1$.

2. Asymptotic Expansions for $H(z)$. As is explained in [8], see also [7], [9], whether or not one can apply the $T$-transformation successfully to the partial sums of the infinite series in (1.6), to obtain good approximations to $H(z)$, depends on whether $H(z)$ satisfies a relation of the form

$$
H(z)=\sum_{i=1}^{n-1} \frac{\mu_{i}}{z^{i}}+R_{n} f(n),
$$

where $R_{n}$ depend on the moments, and $f(y)$, as a function of the continuous variable $y$, has an asymptotic expansion of the form

$$
f(y) \sim \sum_{i=0}^{\infty} \frac{\beta_{i}}{y^{i}} \quad \text { as } y \rightarrow \infty,
$$

and is an infinitely differentiable function of $y$ up to and including $y=\infty$.

In a recent work, [10], the present author has shown that for $w(x)=x^{\alpha} e^{-x}$, $\alpha>-1,(2.1)$ is satisfied with

$$
\begin{gathered}
\mu_{i}=(\alpha+i-1) !, \quad i=1,2, \ldots, \\
R_{n}=\frac{(\alpha+n-1) !}{n z^{n}}
\end{gathered}
$$

and

$$
f(y) \sim \sum_{i=0}^{\infty} \frac{-z e^{-z} \delta_{i}(z)}{y^{i}} \quad \text { as } y \rightarrow \infty,
$$


where

$$
\delta_{i}(z)=\left.\frac{\partial^{i}}{\partial \xi^{i}}\left[e^{(1-\alpha) \xi} \exp \left(z e^{\xi}\right)\right]\right|_{\xi=0}, \quad i=0,1, \ldots
$$

Furthermore, (2.1) holds for all $z \notin[0, \infty)$.

In [10] it is shown that also for $w(x)=x^{\alpha} E_{p}(x), \alpha>-1, p+\alpha>0$, where $E_{p}(x)=\int_{1}^{\infty}\left(e^{-x t} / t^{p}\right) d t$ is the exponential integral, whenever $\operatorname{Re} z<0$, (2.1) is again satisfied with

$$
\mu_{i}=\frac{(\alpha+i-1) !}{p+\alpha+i-1}, \quad i=1,2, \ldots,
$$

and $R_{n}$ exactly as given in (2.4), and $f(y)$ given by

$$
f(y) \sim \sum_{i=0}^{\infty} \frac{-z \varepsilon_{i}(z)}{y^{i}} \text { as } y \rightarrow \infty
$$

where

$$
\varepsilon_{i}(z)=\left.\frac{\partial^{i}}{\partial \xi^{i}}\left[e^{(1-\alpha) \xi} \exp \left(z e^{\xi}\right) \int_{0}^{\xi} d \sigma e^{(1-p) \sigma} \exp \left(-z e^{\sigma}\right)\right]\right|_{\xi=0}, \quad i=0,1, \ldots
$$

Although the truth of (2.1) and (2.2) for this case has been shown only for $\operatorname{Re} z<0$, we believe that they hold also for $\operatorname{Re} z \geqslant 0$.

For the details of the computations that lead to the results above, see [10].

\section{Derivation of Numerical Quadrature Formulas and Some Properties. We now} take up the problem of derivation of the new numerical quadrature formulas $J_{k}[f]$ for the infinite range integrals $J[f]$ (see (1.4)) for the weight functions $w(x)=$ $x^{\alpha} e^{-x}, \alpha>-1$, and $w(x)=x^{\alpha} E_{p}(x), \alpha>-1, p+\alpha>0$. Let $A_{0}=0$ and $A_{r}=$ $\sum_{i=1}^{r} \mu_{i} / z^{i}, r=1,2, \ldots$, and $R_{r}=\left[(\alpha+r-1) ! / z^{r}\right] r^{-1}, r=1,2, \ldots$ Applying the modified version of the $T$-transformation (see Eq. (2.2) in [8]), namely,

$$
T_{k, n}=\frac{\sum_{j=0}^{k}(-1)^{j}\left(\begin{array}{l}
k \\
j
\end{array}\right)(n+j)^{k-1} A_{n+j-1} / R_{n+j}}{\sum_{j=0}^{k}(-1)^{j}\left(\begin{array}{l}
k \\
j
\end{array}\right)(n+j)^{k-1} / R_{n+j}},
$$

to the sequence above, and cancelling a factor of $z$ from the numerator and the denominator, we obtain

$$
T_{k, n}=\frac{\sum_{j=0}^{k}(-1)^{j}\left(\begin{array}{l}
k \\
j
\end{array}\right) \frac{(n+j)^{k}}{(\alpha+n+j-1) !} z^{n+j-1} A_{n+j-1}}{\sum_{j=0}^{k}(-1)^{j}\left(\begin{array}{l}
k \\
j
\end{array}\right) \frac{(n+j)^{k}}{(\alpha+n+j-1) !} z^{n+j-1}} .
$$

$T_{k, n}$ is an approximation to $H(z)$ in (1.5), obtained from the first $n+k-1$ terms of its asymptotic series (1.6). It can easily be verified that $T_{k, n}$ is a rational function in $z$ whose numerator $N_{k, n}(z)$ is a polynomial of degree $\leqslant n+k-2$ and whose denominator $D_{k, n}(z)$ is $z^{n-1}$ times another polynomial of degree exactly $k$, so that (degree of denominator) $\geqslant$ (degree of numerator) +1 , a property that $H_{k}(z)$ must have (see (1.5)). $H_{k}(z)$ is also required to have simple poles, otherwise the numerical 
quadrature formula contains derivatives of $f(x)$ as well as $f(x)$ itself, which is undesirable. Consequently, we must deal with the cases $n=1,2$ exclusively (the cases $n=3,4, \ldots$, give rise to a multiple pole at $z=0$ ). Therefore, it seems that we could set $H_{k}(z)=T_{k, 1}$ or $H_{k}(z)=T_{k-1,2}$ and use these $H_{k}(z)$ to derive numerical quadrature formulas $J_{k}[f]$ for the integral $J[f]$. For example, for $n=1$, for which we give numerical results in this paper, we have

$$
H_{k}(z)=T_{k, 1}=\frac{\sum_{j=0}^{k}(-1)^{j}\left(\begin{array}{l}
k \\
j
\end{array}\right) \frac{(j+1)^{k}}{(\alpha+j) !} z^{j} A_{j}}{\sum_{j=0}^{k}(-1)^{j}\left(\begin{array}{l}
k \\
j
\end{array}\right) \frac{(j+1)^{k}}{(\alpha+j) !} z^{j}}=\frac{N_{k, 1}(z)}{D_{k, 1}(z)} .
$$

Then the abscissas $x_{k, i}$ are the zeros of the denominator of $T_{k, 1}$ (which are simple as we shall show below), and the weights $A_{k, i}$ are the residues of $T_{k, 1}$ at the corresponding $x_{k, i}$, i.e.,

$$
A_{k, i}=\left.\frac{N_{k, 1}(z)}{\frac{d}{d z} D_{k, 1}(z)}\right|_{z=x_{k, i}}, \quad i=1, \ldots, k .
$$

Remark 1. Since the denominators of $T_{k, 1}$ and $T_{k-1,2}$ depend only on $\alpha$ and not on $p$, their zeros, therefore, the abscissas $x_{k, i}$ of our new numerical quadrature formulas, are independent of $p$. Consequently, we have the same set of abscissas for the weight functions $w_{1}(x)=x^{\alpha} e^{-x}$ and $w_{2}(x)=x^{\alpha} E_{p}(x), \alpha>-1, p+\alpha>0$. Furthermore, the polynomials that provide us with the abscissas are known analytically. This is not the case for the Gaussian formulas for the weight function $w_{2}(x)$.

Remark 2. The advantage of the new formulas mentioned in Remark 1 are a consequence of our flexible modification of the $T$-transformation of Levin. If Levin's $t$ - (or $u$-) transformation is used to obtain the new numerical quadrature formulas, then, since $R_{n}=\mu_{n} / z^{n}$ (or $R_{n}=n \mu_{n} / z^{n}$ ), this advantage disappears altogether.

We demand of a good numerical quadrature formula that its abscissas be in the interval of integration. For the formulas obtained from $H_{k}(z)=T_{k, 1}$ and $H_{k}(z)=$ $T_{k-1,2}$ we can show that this holds true.

THEOREM 3.1. Let

$$
D_{k, n, m}(z)=\sum_{j=0}^{k}(-1)^{j}\left(\begin{array}{l}
k \\
j
\end{array}\right) \frac{(n+j)^{m}}{(\alpha+n+j-1) !} z^{n+j-1},
$$

where $m$ is a nonnegative integer. Then

$$
D_{k, n, m}(z)=\frac{k !}{(\alpha+n+k-1) !}\left(\frac{d}{d z} z\right)^{m}\left[z^{n-1} L_{k}^{(\alpha+n-1)}(z)\right],
$$

where $L_{k}^{(s)}(z)$ is the generalized Laguerre polynomial of degree $k$. Furthermore, $D_{k, n, m}(z)$ has a zero of multiplicity $n-1$ at $z=0$ and $k$ simple zeros in $(0, \infty)$ and between two simple zeros of $D_{k, n, m}(z)$ there is exactly one simple zero of $D_{k, n, m-1}(z)$, and the first positive zero of $D_{k, n, m}(z)$ is smaller than the first positive zero of $D_{k, n, m-1}(z)$. 
Remark. Note that the case of interest in this work is $m=k$.

Proof. (3.6) follows from (3.5) by observing that

$$
D_{k, n, m}(z)=\left(\frac{d}{d z} z\right)^{m} \sum_{j=0}^{k}(-1)^{j}\left(\begin{array}{l}
k \\
j
\end{array}\right) \frac{z^{n+j-1}}{(\alpha+n+j-1) !}
$$

and identifying the summation on the right-hand side of (3.7) as $C_{k, n} z^{n-1} L_{k}^{(\alpha+n-1)}(z)$, where $C_{k, n}=k ! /(\alpha+n+k-1)$ !, see Abramowitz and Stegun [1, p. 775]. From (3.6) it follows that

$$
D_{k, n, m+1}(z)=\frac{d}{d z}\left[z D_{k, n, m}(z)\right], \quad m=0,1, \ldots
$$

The rest of the theorem can now be proved by induction. Set $m=0$. First $D_{k, n, 0}(z)=C_{k, n} z^{n-1} L_{k}^{(\alpha+n-1)}(z)$, has a zero of multiplicity $n-1$ at $z=0$ and $k$ simple zeros $x_{i}^{(0)}, i=1, \ldots, k$, in $(0, \infty)$ which we order as $0<x_{1}^{(0)}<x_{2}^{(0)}$ $<\cdots<x_{k}^{(0)}$, namely those of $L_{k}^{(\alpha+n-1)}(z)$. Therefore, $D_{k, n, 1}(z)=$ $(d / d z)\left[z D_{k, n, 0}(z)\right]$, by Rolle's theorem, has a zero of multiplicity $n-1$ at $z=0$ and $k$ simple zeros $x_{i}^{(1)}, i=1, \ldots, k$, such that $0<x_{1}^{(1)}<x_{1}^{(0)}<x_{2}^{(1)}<x_{2}^{(0)}<\cdots<$ $x_{k}^{(1)}<x_{k}^{(0)}$. The rest of the proof for general $m$ is now obvious.

By letting $m=k$ in Theorem 3.1 we have the following result.

COROLlary. $T_{k, 1}$ has $k$ simple poles in $(0, \infty)$ and $T_{k-1,2}$ has a simple pole at $z=0$ and $k-1$ simple poles in $(0, \infty)$; i.e., the abscissas of the numerical quadrature formulas obtained by setting $H_{k}(z)=T_{k, 1}\left(\right.$ or $\left.H_{k}(z)=T_{k-1,2}\right)$ are distinct, real and in $(0, \infty)$ (or in $[0, \infty)$ with $z=0$ being one of them).

TheOREM 3.2. Let $\sigma_{j}, j=0,1,2, \ldots$, be constants independent of $k$, and define

$$
G_{k, n, m}(z)=\sum_{j=0}^{k}\left(\begin{array}{l}
k \\
j
\end{array}\right) \sigma_{j}(n+j)^{m} z^{n+j-1}=\left(\frac{d}{d z} z\right)^{m} \sum_{j=0}^{k}\left(\begin{array}{l}
k \\
j
\end{array}\right) \sigma_{j} z^{n+j-1}
$$

Then

$$
G_{k, n, m}(z)=(n+k) G_{k, n, m-1}(z)-k G_{k-1, n, m-1}(z) .
$$

Proof. We can express (3.9) in the form

$$
\begin{aligned}
G_{k, n, m}(z) & =\left(\frac{d}{d z} z\right)^{m-1} \frac{d}{d z} \sum_{j=0}^{k}\left(\begin{array}{l}
k \\
j
\end{array}\right) \sigma_{j} z^{n+j} \\
& =\left(\frac{d}{d z} z\right)^{m-1} \sum_{j=0}^{k}\left(\begin{array}{l}
k \\
j
\end{array}\right) \sigma_{j}[(n+k)-(k-j)] z^{n+j-1} .
\end{aligned}
$$

The result in (3.10) now follows by noting that $\left(\begin{array}{c}k \\ j\end{array}\right)(k-j)=k\left(\begin{array}{c}k-1 \\ j\end{array}\right)$ for $j=$ $0, \ldots, k-1$ and 0 for $j=k$.

COROLlary. The polynomials $D_{k, n, m}(z)$ satisfy

$$
D_{k, n, m}(z)=(n+k) D_{k, n, m-1}(z)-k D_{k-1, n, m-1}(z) .
$$

Proof. (3.12) follows from the fact that $D_{k, n, m}(z)$ are polynomials of the form of $G_{k, n, m}(z)$ in Theorem 3.2 above with $\sigma_{j}=(-1)^{j} /(\alpha+n+j-1) !, j=0,1, \ldots$

Making use of Theorem 3.2, we can prove that the abscissas of our new numerical quadrature formulas have the interlacing property like those of the Gaussian quadrature formulas. 
THEOREM 3.3. Let $x_{1}^{k, m}<x_{2}^{k, m}<\cdots<x_{k}^{k, m}$ be the simple zeros of $D_{k, n, m}(z)$ in $(0, \infty)$ (see Theorem 3.1). Then between two consecutive such zeros of $D_{k, n, m}(z)$ there is exactly one simple zero of $D_{k-1, n, m-1}(z), m \geqslant 2$.

Proof. If we let $z=x_{j}^{k, m}$ and $z=x_{j+1}^{k, m}, 1 \leqslant j \leqslant k-1$ in (3.12) and use the fact that $D_{k, n, m}\left(x_{i}^{k, m}\right)=0, i=j, j+1$, we obtain

$$
k D_{k-1, n, m-1}\left(x_{i}^{k, m}\right)=(n+k) D_{k, n, m-1}\left(x_{i}^{k, m}\right), \quad i=j, j+1 .
$$

In Theorem 3.1 we showed that between two simple zeros of $D_{k, n, m}(z)$ in $(0, \infty)$ there is exactly one simple zero of $D_{k, n, m-1}(z)$. Therefore, $D_{k, n, m-1}\left(x_{i}^{k, m}\right) \neq 0$, $i=1,2, \ldots, k$, furthermore $D_{k, n, m-1}\left(x_{j}^{k, m}\right)$ and $D_{k, n, m-1}\left(x_{j+1}^{k, m}\right)$ have opposite signs. This together with (3.13) implies that $D_{k-1, n, m-1}\left(x_{j}^{k, m}\right)$ and $D_{k-1, n, m-1}\left(x_{j+1}^{k, m}\right)$ have opposite signs too. Therefore, $D_{k-1, n, m-1}(z)$ must vanish at least once in $\left(x_{j}^{k, m}, x_{j+1}^{k, m}\right)$. But since the number of simple zeros of $D_{k-1, n, m-1}(z)$ on $(0, \infty)$ is $k-1$, there can be at most one zero in $\left(x_{j}^{k, m}, x_{j+1}^{k, m}\right)$. This completes the proof of the theorem.

COROLlaRY. The positive abscissas of the new numerical quadrature formulas obtained from the rational functions $T_{k, n}$ and $T_{k-1, n}(n=1,2)$ interlace.

Although we have not proved the positiveness of the weights $A_{k, i}$ of our new numerical quadrature formulas, numerical results indicate that they are. This, as is known, is an important property that numerical quadrature formulas are required to have.

Another property of our new formulas is that they are of interpolatory type, see [8], as the following result states.

THEOREM 3.4. Let $J_{k}[f]$ in (1.4) be the numerical quadrature formula associated with $H_{k}(z)=T_{k, 1}$ or $H_{k}(z)=T_{k-1,2}$. Then

$$
J_{k}\left[x^{i}\right]=J\left[x^{i}\right], \quad i=0,1, \ldots, k-1,
$$

both for $w_{1}(x)=x^{\alpha} e^{-x}$ and $w_{2}(x)=x^{\alpha} E_{p}(x), \alpha>-1, p+\alpha>0$, and

$$
J_{k}\left[x^{i}\right]=J\left[x^{i}\right], \quad i=0,1, \ldots, k,
$$

for $w_{2}(x)$ whenever $p+\alpha=1$.

Proof. The same as that of Theorem 4.1 and the remark following it in [8].

Remark 3. Theorems 3.1 and 3.3 and their corollaries are similar in nature to the corresponding theorems in [8]. The result of Theorem 3.2, however, is new and contains Eq. (4.14) in [8] as a special case.

4. Numerical Results. In this section we shall give some numerical results obtained by taking $H_{k}(z)=T_{k, 1}$. The results for $H_{k}(z)=T_{k-1,2}$ are about the same as those for $H_{k}(z)=T_{k, 1}$ (they both are $k$-point formulas) and will not be given here. 


\section{TABle 4.1a}

The abscissas $x_{k, i}$ for the new numerical quadrature formulas $\int_{0}^{\infty} \omega(x) f(x) d x \approx$ $\sum_{i=1}^{k} A_{k, i}\left(x_{k, i}\right)$ where $w(x)=x^{\alpha} e^{-x}$ or $w(x)=x^{\alpha} E_{p}(x), p>0$. These $x_{k, i}$ are the roots of the polynomial equation $\sum_{j-0}^{k} \lambda_{j} z^{j}=0$ with $\lambda_{j}=(-1)^{j}\left(\begin{array}{l}k \\ j\end{array}\right)(j+1)^{k} / j !, j=0,1, \ldots, k$. The weights $A_{k, i}$ can be computed from (4.2).

$$
K=2
$$

$0.1352966685416368734372715 Q+00$ $0.1642481109236140904340506 Q+01$

$$
K=3
$$

$0.4505096041273612628731663 Q-01$ $0.6767167970341259016621497 Q+00$ $0.3075107242553137972050534 Q+01$

$$
K=4
$$

$0.1666747355483805876646528 Q-01$ $0.3180181842758116537606890 Q+00$ $0.15524710131218958770477650+01$ $0.4666443329047454410425080 Q+01$

$0.65752864613535943365865200-02$ $0.1606142317401454028672786 Q+j 0$ $0.8655532227468952548875133 Q+00$ $0.2654640475454163235703710 Q+01$ $0.6359556084008965145949768 Q+01$

$K=6$

$0.2707864701816735328121392 Q-02$ $0.8508305148428640858724367 Q-01$ $0.5092057433688204133906909 Q+00$ $0.1639972558714133179393992 Q+01$ $0.3915248557178793725173108 Q+01$ $0.81242826622949267823023150+01$

$K=7$

$0.11497914485285183229071990-02$ $0.4664783979294682653256681 Q-01$ $0.3106265453447279364212658 Q+00$ $0.1058211225813305334731752 Q+01$ $0.2590541885281939341616039 Q+01$ $0.5292380339780669851059954 Q+01$ $0.9942541657663614613190516 Q+01$

$K=8$

$0.4994312298446427845065726 Q-03$ $0.26249621910425722940990590-01$ D. $1946430851588193647017747 Q+00$ $0.7030609671769538287123412 Q+00$ $0.1779188598644186365876674 Q+01$ $0.3678545091181902027940886 Q+01$ $0.6758890871288327828074605 Q+01$ $0.1180256029366207606252295 Q+02$
$K=9$

$0.2207521317724925858938488 Q-03$ $0.1507552393251116722526709 Q-01$ $0.1245393110961793261601531 Q+00$ $0.4773846078371063555094626 Q+00$ $0.12525238413602542256205650+01$ $0.2642940899971808063081664 Q+01$ $0.4875885820583371555261397 Q+01$ $0.8296284357470376096847307 Q+01$ $0.1369620449461662071770829 Q+02$

$K=10$

$0.9892395934261595489626847 Q-04$ $0.8801264957863189992681821 Q-02$ $0.8103177761469819944716406 Q-01$ $0.3297575418975984320679508 Q+00$ $0.8980931136795896724077270 Q+00$ $0.19404978203662116331960740+01$ $0.3624993110444317167348184 Q+01$ $0.61620580548228508707459520+01$ $0.9891400587100293449508436 Q+01$ $0.15617596748110409505771300+02$

$$
K=11
$$

$0.44823203426575744895671330-04$ $0.5207909623162271469395125 Q-02$ $0.5345475360211059413823949 Q-01$ $0.2309906558108135273776385 Q+00$ $0.6533678661614567980364082 Q+00$ $0.1447701829626337013453597 Q+01$ $0.27481048927326722791116290+01$ $0.4705876379525167924577051 Q+01$ $0.7521799853246242986333288 Q+01$ $0.1153453533217644513798817 Q+02$ $0.1756233860215826167109416 Q+02$

$$
K=12
$$

$0.20494814735885028389632310-04$ $0.3116434971088132598181122 Q-02$ $0.35671293788086112260485810-01$ $0.1637038106438552243872225 Q+00$ $0.48102263368462321873361230+00$ $0.10937497897582915613546130+01$ $0.2113272551481096111677349 Q+01$ $0.3658647547020350981572865 Q+01$ $0.5870258102208375884709533 Q+01$ $0.8943499910675444211687219 Q+01$ $0.1321831883068720803236893 Q+02$ $0.19527044361767893008995420+02$ 
TABLE 4.1b

The weights $A_{k, i}$ for the new numerical quadrature formulas $\int_{0}^{\infty} e^{-x} f(x) d x \approx \sum_{i=1}^{k} A_{k, i} f\left(x_{k, i}\right)$. The $x_{k, i}$ are given in Table 4.1a.

$$
K=2
$$

$0.4262790219225514332710374 Q+00$ $0.5737209780774485667289626 Q+00$

$K=3$

$0.1719726235072209047509659 Q+00$ $0.6479430891839743129379293 Q+00$ $0.1800842873088047823111049 Q+00$

$$
K=4
$$

$0.7226231915410304044686545 Q-01$ $0.4675117155079844345906701 Q+00$ $0.4166703847548192995002485 Q+00$ $0.43555580583093225462215960-01$

$$
K=5
$$

$0.3162835785818733939584087 Q-01$ $0.2964059964296510953358956 Q+00$ $0.4836094609514361588023003 Q+00$ $0.1792932701658073980412668 Q+00$ $0.9062914594918008424696461 Q-02$

$$
K=6
$$

$0.1428211335995379924913036 Q-01$ $0.1810029734952154472350761 Q+00$ $0.4283183120895699928286281 Q+00$ $0.3139891339858145485368878 Q+00$ $0.6070052264960922735114748 \mathrm{Q}-01$ $0.1706944419836984799130211 Q-02$

$$
K=7
$$

$0.6598330798239452961485086 Q-02$ $0.1098248922206528668845921 Q+00$ $0.3358101635284652760831161 Q+00$ $0.37713117010376235364611230+00$ $0.1528537231889653157956051 Q+00$ $0.1748243331749687194914008 Q-01$ $0.29928684241786267994914780-03$

$$
K=8
$$

$0.3099870283959833613571391 Q-02$ $0.6693866681287097397256413 Q-01$ $0.2485362003123865502072833 Q+00$ $0.3727396542211409727822777 Q+00$ $0.2431885810309434298663758 Q+00$ $0.6097027090259757966354025 Q-01$ $0.4477052871576393867391677 Q-02$ $0.4970356452426602699576381 Q-04$
$K=9$

$0.14744694007014387646032640-02$ $0.41127429883070365438311820-01$ $0.17902009302818061702374070+00$ $0.33068623673791203980016690+00$ $0.2998224185517629141010072 Q+00$ $0.12582632321379700001159620+00$ $0.20987060228646790681414680-01$ $0.10480601328389181946816570-02$ $0.7908823089915984477495928 Q-05$

$$
K=10
$$

$0.70790228354985238418887130-03$ $0.25488325595118355474619360-0$ $0.1274226523339195729573121 Q+00$ $0.2757347080459756551452594 Q+00$ $0.31729741898747736645572720+00$ $0.1915255678928506327037948 Q+00$ $0.5514876022240948056329139 Q-01$ $0.6444963104831028723924859 Q-02$ $0.2284860115590542510026685 Q-03$ $0.1215522309001340879340377 Q-05$

$$
K=11
$$

$0.3422920264581562223112103 Q-03$ $0.1592627133604990388353136 Q-01$ $0.9032697757481774988251322 Q-01$ $0.2217346103387849083743623 Q+00$ $0.3052359123969847078321431 Q+00$ $0.2407992784200027993700258 Q+00$ $0.1025645532644478690600888 Q+00$ $0.2121654709984841223948429 Q-01$ $0.1806373139670102934608411 Q-02$ $0.4700289257745581260633884 Q-04$ $0.1815103579343883250561201 Q-06$

$$
K=12
$$

$0.1664210817022919684179579 Q-03$ $0.1002537753936819105781108 Q-01$ $0.6402700468555379941006606 Q-01$ $0.1745721822360148306828806 Q+00$ $0.2762012799800637007904487 Q+00$ $0.2670162411605854677879026 Q+00$ $0.15244363583178747681119740+00$ $0.47724514191716621639404870-01$ $0.7344423584282093647907940 Q-02$ $0.4696806037099412766549128 Q-03$ $0.92126530298247906310653640-05$ $0.26452185760136677218286860-07$ 
TABLE 4.1c

The weights $A_{k, i}$ for the new numerical quadrature formulas $\int_{0}^{\infty} E_{1}(x) f(x) d x \approx$ $\sum_{i=1}^{k} A_{k, i} f\left(x_{k, i}\right)$. The $x_{k, i}$ are given in Table 4.1a.

$K=2$

$0.7580234232710699835513690 Q+00$ $0.2419765767289300164486310 Q+00$

$K=3$

$0.4554540735108335506704450 Q+00$ $0.4982740688105893984787207 Q+00$ $0.4627185767857705085083424 \mathrm{Q}-01$

$K=4$

$0.2559124314488475647034520 Q+00$ $0.5540725756664822258223339 Q+00$ $0.1821354548398085887398495 Q+00$ $0.7879538044861620734364579 Q-02$

$K=5$

$0.1402073401311772741711620 Q+00$ $0.4891155383497700586633836 Q+00$ $0.3175517522863648430356046 Q+00$ $0.5187500819320468386324305 Q-01$ $0.1250361039483140266606738 Q-02$

$k=6$

$0.7590679384919516304074620 Q-01$ $0.3882569073353100374747409 Q+00$ $0.3910982789957450034559817 Q+00$ $0.1317949666122117178283279 Q+00$ $0.1275405893635085165625092 Q-01$ $0.1889942711872265439523386 Q-03$

$K=7$

$0.4081165699922444055525246 Q-01$ $0.2916484563421986709994837 Q+00$ $0.4031742897215124443059980 Q+00$ $0.216 .4039357580742197306810 Q+00$ $0.4509850270751601688195192 Q-01$ $0.2835608913318645418197694 Q-02$ $0.2754955815556210843516167 Q-04$

$K=8$

$0.2183694095237601731347362 Q-01$ $0.2123436066706023280859898 Q+00$ $0.3759015998592184903536997 Q+00$ $0.2797841689691778014757056 Q+00$ $0.96044317563684141994846530-01$ $0.1350041606147886266984887 Q-01$ $0.5850466653776554364622037 Q-03$ $0.3903258084702669973656278 Q-05$
$0.11639481487006604333914510-01$ $0.1517165526134532491022856 Q+00$ $0.3298341874710617676412120 Q+00$ $0.3135048910910120987495115 Q+00$ $0.1530007866011305814318330 Q+00$ $0.36534277886046047529808790-01$ $0.3655427407496274166283388 Q-02$ $0.1138550570413118897646681 Q-03$ $0.5403857520651553866290788 Q-06$

$$
K=10
$$

$0.6183607395711354532866023 Q-02$ $0.10710911018860638895497070+00$ $0.2784453407952368949679836 Q+00$ $0.32080169090502123758203610+00$ $0.20333975793571523682184970+00$ $0.7082269642288027252226315 Q-01$ $0.1236216945865997469233175 Q-01$ $0.91442110008256518269362160-03$ $0.2113241163514073803417835 Q-04$ $0.7338645093400497129421534 Q-07$

$$
K=11
$$

$0.32753845301042776702932810-02$ $0.7501957741413657025016744 Q-01$ $0.2291117291836910307454911 Q+00$ $0.3090943534216158917513319 Q+00$ $0.23984222788693268973962400+00$ $0.1107670005330865349182265 Q+00$ $0.2886106586082509163584454 \mathrm{Q}-01$ $0.38104387417493704678944370-02$ $0.2144416921941403642591808 Q-03$ $0.3770931329409887434130059 Q-05$ $0.9804334992569433381436212 Q-08$

$$
K=12
$$

$0.1730217229647563323727468 Q-02$ $0.5225852598266425798596882 \mathrm{Q}-01$ $0.1852238054344086666606745 \mathrm{Q}+00$ $0.28575797362654439881135410+00$ $0.2607043547367742704644881 Q+00$ $0.1497818572726624001050412 Q+00$ $0.5279170686778839885617192 Q-01$ $0.1061556042946016328951724 Q-01$ $0.1037699857196495210286065 \mathrm{Q}-02$ $0.4764646687998658382098453 Q-04$ $0.6508045093724211033391325 Q-06$ $0.1291464026287846155793969 Q-08$ 
TABLE 4.2

$S_{k}$ and $G_{k}$ stand for the $k$-point new rule and Gauss-Laguerre rule respectively, with $w(x)=$ $e^{-x}$. The abscissas for $S_{k}$ are those given in Table 4.1a, and the corresponding weights are given in Table 4.1b.

\begin{tabular}{|l|l|l|l|l|l|l|}
\hline \multirow{2}{*}{ Rule } & \multicolumn{7}{|c|}{$\mid J\left[f j-J_{k}[f] \mid\right.$ for J[f] $\int^{\infty} e^{-x} f(x) d x$} \\
\cline { 2 - 8 } & $f(x)=\frac{1}{1+x^{2}}$ & $f(x)=\frac{1}{4+x^{2}}$ & $f(x)=\frac{1}{e^{x}+1}$ & $f(x)=\frac{1}{1+x}$ & $f(x)=\frac{1}{2+x}$ & $f(x)=e^{-x}$ \\
\hline$S_{2}$ & $5 \times 10^{-2}$ & $8 \times 10^{-3}$ & $2 \times 10^{-4}$ & $4 \times 10^{-3}$ & $4 \times 10^{-3}$ & $2 \times 10^{-2}$ \\
$G_{2}$ & $3 \times 10^{-2}$ & $6 \times 10^{-3}$ & $3 \times 10^{-3}$ & $3 \times 10^{-2}$ & $4 \times 10^{-3}$ & $2 \times 10^{-2}$ \\
\hline$S_{4}$ & $5 \times 10^{-4}$ & $1 \times 10^{-3}$ & $9 \times 10^{-4}$ & $4 \times 10^{-4}$ & $1 \times 10^{-5}$ & $1 \times 10^{-4}$ \\
$G_{4}$ & $2 \times 10^{-2}$ & $3 \times 10^{-4}$ & $2 \times 10^{-5}$ & $3 \times 10^{-3}$ & $2 \times 10^{-4}$ & $3 \times 10^{-4}$ \\
\hline$S_{6}$ & $2 \times 10^{-3}$ & $1 \times 10^{-4}$ & $1 \times 10^{-4}$ & $2 \times 10^{-5}$ & $2 \times 10^{-6}$ & $1 \times 10^{-5}$ \\
$G_{6}$ & $3 \times 10^{-4}$ & $1 \times 10^{-4}$ & $4 \times 10^{-5}$ & $6 \times 10^{-4}$ & $2 \times 10^{-5}$ & $5 \times 10^{-6}$ \\
\hline$S_{8}$ & $1 \times 10^{-4}$ & $1 \times 10^{-5}$ & $1 \times 10^{-5}$ & $5 \times 10^{-7}$ & $6 \times 10^{-8}$ & $3 \times 10^{-7}$ \\
$G_{8}$ & $1 \times 10^{-3}$ & $1 \times 10^{-5}$ & $4 \times 10^{-6}$ & $1 \times 10^{-4}$ & $3 \times 10^{-6}$ & $7 \times 10^{-8}$ \\
\hline$S_{10}$ & $8 \times 10^{-5}$ & $1 \times 10^{-6}$ & $9 \times 10^{-7}$ & $2 \times 10^{-8}$ & $2 \times 10^{-10}$ & $3 \times 10^{-8}$ \\
$G_{10}$ & $6 \times 10^{-4}$ & $8 \times 10^{-6}$ & $1 \times 10^{-6}$ & $4 \times 10^{-5}$ & $4 \times 10^{-7}$ & $1 \times 10^{-9}$ \\
\hline$S_{12}$ & $2 \times 10^{-6}$ & $5 \times 10^{-7}$ & $9 \times 10^{-9}$ & $3 \times 10^{-9}$ & $1 \times 10^{-10}$ \\
$G_{12}$ & $1 \times 10^{-4}$ & $8 \times 10^{-7}$ & $2 \times 10^{-7}$ & $1 \times 10^{-5}$ & $1 \times 10^{-7}$ \\
$1 \times 10^{-11}$ \\
\hline$E_{10 a c t}$ & $0.62144 \ldots$ & $0.19951 \ldots$ & $0.30685 \ldots$ & $0.59634 \ldots$ & $0.36132 \ldots$ & 0.5 \\
\hline
\end{tabular}

The abscissas $x_{k, i}$ for the weight functions $w_{1}(x)=x^{\alpha} e^{-x}$ and $w_{2}(x)=x^{\alpha} E_{p}(x)$, $\alpha>-1, p+\alpha>0$, have been determined for different values of $\alpha$ and those for $\alpha=0, k=2(1) 12$, are given in Table 4.1a. Recall that for a fixed value of $\alpha, w_{1}(x)$ and $w_{2}(x)$ (for all $p$ such that $\alpha+p>0$ ) have the same set of abscissas obtained by solving the polynomial equation $\sum_{j=0}^{k} \lambda_{j} z^{j}=0$, where

$$
\lambda_{j}=(-1)^{j}\left(\begin{array}{l}
k \\
j
\end{array}\right) \frac{(j+1)^{k}}{(\alpha+j) !}, \quad j=0,1, \ldots, k .
$$

Once the abscissas $x_{k, i}$ have been computed, the corresponding weights $A_{k, i}$ can be determined from the formula

$$
A_{k, i}=\left.\frac{\sum_{j=0}^{k} \lambda_{j} z^{j} A_{j}}{\sum_{j=0}^{k} j \lambda_{j} z^{j-1}}\right|_{z=x_{k, i}}, \quad i=1, \ldots, k .
$$

In Table 4.2 we compare the approximations $J_{k}[f]$ obtained by using the new numerical quadrature formulas and the corresponding Gauss-Laguerre rules with $w(x)=e^{-x}$. The abscissas for the new rules are those given in Table 4.1a, and the 


\section{TABLE 4.3}

$S_{k}$ and $G_{k}$ stand for the $k$-point new rule and Gauss-Laguerre rule respectively with $w(x)=$ $e^{-x}$, i.e., the singularities of the integrands $f(x)$ at $x=0$ are ignored. The abscissas for $S_{k}$ are thase given in Table 4.1a, and the corresponding weights are given in Table 4.1b.

\begin{tabular}{|c|c|c|c|c|c|}
\hline \multirow{2}{*}{ Rule } & \multicolumn{5}{|c|}{$\left|J[f]-J_{k}[f]\right|$ for $J[f]=\int_{0}^{\infty} e^{-x} f(x) d x$} \\
\hline & $f(x)=x^{-\frac{1}{2}}$ & $f(x)=\log x$ & $f(x)=x^{\frac{1}{2}} \log x$ & $f(x)=x^{\frac{1}{2}}$ & $f(x)=x^{3 / 2}$ \\
\hline $\begin{array}{l}S_{2} \\
G_{2}\end{array}$ & $\begin{array}{l}2 \times 10^{-1} \\
6 \times 10^{-1}\end{array}$ & $\begin{array}{l}9 \times 10^{-3} \\
3 \times 10^{-1}\end{array}$ & $\begin{array}{l}2 \times 10^{-2} \\
5 \times 10^{-2}\end{array}$ & $\begin{array}{l}6 \times 10^{-3} \\
4 \times 10^{-2}\end{array}$ & $\begin{array}{l}1 \times 10^{-1} \\
2 \times 10^{-2}\end{array}$ \\
\hline $\begin{array}{l}S_{4} \\
G_{4}\end{array}$ & $\begin{array}{l}3 \times 10^{-2} \\
4 \times 10^{-1}\end{array}$ & $\begin{array}{l}4 \times 10^{-3} \\
2 \times 10^{-1}\end{array}$ & $\begin{array}{l}7 \times 10^{-4} \\
3 \times 10^{-2}\end{array}$ & $\begin{array}{l}1 \times 10^{-6} \\
1 \times 10^{-2}\end{array}$ & $\begin{array}{l}3 \times 10^{-4} \\
3 \times 10^{-3}\end{array}$ \\
\hline $\begin{array}{l}S_{6} \\
G_{6}\end{array}$ & $\begin{array}{l}8 \times 10^{-4} \\
3 \times 10^{-1}\end{array}$ & $\begin{array}{l}6 \times 10^{-4} \\
1 \times 10^{-1}\end{array}$ & $\begin{array}{l}8 \times 10^{-4} \\
2 \times 10^{-2}\end{array}$ & $\begin{array}{l}3 \times 10^{-5} \\
7 \times 10^{-3}\end{array}$ & $\begin{array}{l}2 \times 10^{-7} \\
1 \times 10^{-3}\end{array}$ \\
\hline $\begin{array}{l}\mathrm{S}_{8} \\
\mathrm{G}_{8}\end{array}$ & $\begin{array}{l}3 \times 10^{-3} \\
3 \times 10^{-1}\end{array}$ & $\begin{array}{l}1 \times 10^{-4} \\
8 \times 10^{-2}\end{array}$ & $\begin{array}{l}3 \times 10^{-5} \\
1 \times 10^{-2}\end{array}$ & $\begin{array}{l}3 \times 10^{-6} \\
5 \times 10^{-3}\end{array}$ & $\begin{array}{l}8 \times 10^{-8} \\
5 \times 10^{-4}\end{array}$ \\
\hline $\begin{array}{l}S_{10} \\
G_{10}\end{array}$ & $\begin{array}{l}2 \times 10^{-3} \\
3 \times 10^{-1}\end{array}$ & $\begin{array}{l}1 \times 10^{-4} \\
6 \times 10^{-2}\end{array}$ & $\begin{array}{l}7 \times 10^{-7} \\
1 \times 10^{-2}\end{array}$ & $\begin{array}{l}9 \times 10^{-7} \\
3 \times 10^{-3}\end{array}$ & $\begin{array}{l}3 \times 10^{-8} \\
3 \times 10^{-4}\end{array}$ \\
\hline $\begin{array}{l}S_{12} \\
G_{12} \\
\end{array}$ & $\begin{array}{l}1 \times 10^{-3} \\
3 \times 10^{-1}\end{array}$ & $\begin{array}{l}2 \times 10^{-5} \\
5 \times 10^{-2}\end{array}$ & $\begin{array}{l}3 \times 10^{-6} \\
8 \times 10^{-3}\end{array}$ & $\begin{array}{l}4 \times 10^{-7} \\
3 \times 10^{-3}\end{array}$ & $\begin{array}{l}4 \times 10^{-9} \\
2 \times 10^{-4}\end{array}$ \\
\hline $\mathrm{G}_{16}$ & $2 \times 10^{-1}$ & $4 \times 10^{-2}$ & $6 \times 10^{-3}$ & $2 \times 10^{-3}$ & $6 \times 10^{-5}$ \\
\hline$G_{24}$ & $2 \times 10^{-1}$ & $3 \times 10^{-2}$ & $3 \times 10^{-3}$ & $9 \times 10^{-4}$ & $3 \times 10^{-5}$ \\
\hline$G_{32}$ & $2 \times 10^{-1}$ & $2 \times 10^{-2}$ & $2 \times 10^{-3}$ & $6 \times 10^{-4}$ & $1 \times 10^{-5}$ \\
\hline Exact & $1.7724 \ldots$ & $-0.57721 \ldots$ & $0.032338 \ldots$ & $0.88622 \ldots$ & $1.3293 .$. \\
\hline
\end{tabular}

corresponding weights are given in Table 4.1b. As can be seen from Table 4.2, for analytic $f(x)$, the new rules compare favorably with the Gaussian rules and in some cases give better results. It is worth noting that, as the singularities of $f(x)$ become farther away from the interval of integration $[0, \infty)$, the $J_{k}[f]$ become better for both rules.

Since the abscissas of the new rules that are given in Table 4.1 do not include the lower limit $x=0$ (a property shared by the Gaussian rules too), we can use the new rules for obtaining approximations to integrals for which $f(x)$ are singular at 
TABLE 4.4

$S_{k}$ and $G_{k}$ stand for the $k$-point new rule and Gaussian rule respectively with $w(x)=E_{1}(x)$. The abscissas for $S_{k}$ are thase given in Table 4.1a, and the corresponding weights are given in Table 4.1c. The abscissas and weights for $G_{10}$ have been taken from the 14-digit tables of Danloy [4].

\begin{tabular}{|c|c|c|c|c|c|c|}
\hline \multirow{2}{*}{ Rule } & \multicolumn{5}{|c|}{$\left|J[f]-J_{k}[f]\right|$ for $J[f]=\int_{0}^{\infty} E_{1}(x) f(x) d x$} \\
\cline { 2 - 8 } & $f(x)=\frac{1}{1+x^{2}}$ & $f(x)=\frac{1}{4+x^{2}}$ & $f(x)=\frac{1}{e^{x}+1}$ & $f(x)=\frac{1}{1+x}$ & $f(x)=\frac{1}{2+x}$ & $f(x)=e^{-x}$ \\
\hline$S_{2}$ & $2 \times 10^{-3}$ & $3 \times 10^{-3}$ & $4 \times 10^{-4}$ & $1 \times 10^{-2}$ & $3 \times 10^{-3}$ & $2 \times 10^{-2}$ \\
\hline$S_{4}$ & $5 \times 10^{-3}$ & $3 \times 10^{-4}$ & $8 \times 10^{-5}$ & $2 \times 10^{-4}$ & $2 \times 10^{-5}$ & $3 \times 10^{-4}$ \\
\hline$S_{6}$ & $1 \times 10^{-3}$ & $1 \times 10^{-5}$ & $1 \times 10^{-5}$ & $9 \times 10^{-7}$ & $2 \times 10^{-6}$ & $4 \times 10^{-6}$ \\
\hline$S_{8}$ & $2 \times 10^{-4}$ & $4 \times 10^{-6}$ & $2 \times 10^{-7}$ & $1 \times 10^{-6}$ & $3 \times 10^{-8}$ & $2 \times 10^{-8}$ \\
\hline$S_{10}$ & $3 \times 10^{-5}$ & $1 \times 10^{-6}$ & $2 \times 10^{-7}$ & $1 \times 10^{-7}$ & $1 \times 10^{-9}$ & $2 \times 10^{-10}$ \\
$G_{10}$ & $1 \times 10^{-4}$ & $2 \times 10^{-6}$ & $6 \times 10^{-9}$ & $2 \times 10^{-5}$ & $2 \times 10^{-7}$ & $2 \times 10^{-10}$ \\
\hline$S_{12}$ & $2 \times 10^{-5}$ & $1 \times 10^{-8}$ & $6 \times 10^{-8}$ & $1 \times 10^{-8}$ & $2 \times 10^{-10}$ & $1 \times 10^{-12}$ \\
\hline$E_{10 x t}$ & $0.80759 \ldots$ & $0.22806 \ldots$ & $0.39225 \ldots$ & $0.74519 \ldots$ & $0.41835 \ldots$ & $0.69314 \ldots$ \\
\hline
\end{tabular}

$x=0$, by avoiding the singularity. It turns out that the new formulas for $w(x)=$ $e^{-x}$ work very efficiently on integrals of the form $\int_{0}^{\infty} e^{-x} f(x) d x$, where the functions $f(x)$ have algebraic or logarithmic singularities or products of them at $x=0$, especially when $f(x)$ are continuous at $x=0$. For such integrals, numerous computations have shown that the new rules are superior to the Gaussian rules with $w(x)=e^{-x}$. In Table 4.3 we compare the new rules and the Gaussian rules, with $w(x)=e^{-x}$, for a set of singular functions. We note that as we proceed to the right along this table, the $f(x)$ become less and less singular.

In Table 4.4 and Table 4.5 we compare the new rules and the Gaussian rules with $w(x)=E_{1}(x)$, on the same functions $f(x)$ that appear in Table 4.2 and Table 4.3 respectively. The abscissas for the new rules are again those given in Table 4.1a, and the corresponding weights are given in Table 4.1c. The results for the Gaussian rules in this case have been obtained by using the 10-point and 20-point 14-digit tables of Danloy [4]. The conclusions from this comparison are the same as those for the case $w(x)=e^{-x}$. 
TABLE 4.5

$S_{k}$ and $G_{k}$ stand for the $k$-point new rule and Gaussian rule respectively with $w(x)=E_{1}(x)$; i.e., the singularities of the integrands $f(x)$ at $x=0$ are ignored. The abscissas for $S_{k}$ are those given in Table 4.1a, and the corresponding weights are given in Table 4.1c. The abscissas and weights for $G_{10}$ and $G_{20}$ have been taken from the 14-digit tables of Danloy [4].

\begin{tabular}{|l|l|l|l|l|l|}
\hline \multirow{2}{*}{ Rule } & \multicolumn{5}{|c|}{$\left|J[f]-J_{k}[f]\right|$ for $J[f]=\int_{0}^{\infty} E_{1}(x) f(x) d x$} \\
\cline { 2 - 7 } & $f(x)=x^{-\frac{1}{2}}$ & $f(x)=10 g x$ & $f(x)=x^{\frac{1}{2}} 10 g x$ & $f(x)=x^{\frac{1}{2}}$ & $f(x)=x^{3 / 2}$ \\
\hline$S_{2}$ & $1 \times 10^{0}$ & $2 \times 10^{-1}$ & $3 \times 10^{-2}$ & $2 \times 10^{-3}$ & $2 \times 10^{-2}$ \\
\hline$S_{4}$ & $4 \times 10^{-1}$ & $1 \times 10^{-2}$ & $5 \times 10^{-3}$ & $2 \times 10^{-3}$ & $7 \times 10^{-5}$ \\
\hline$S_{6}$ & $1 \times 10^{-1}$ & $1 \times 10^{-2}$ & $6 \times 10^{-4}$ & $2 \times 10^{-5}$ & $4 \times 10^{-6}$ \\
\hline$S_{8}$ & $8 \times 10^{-3}$ & $1 \times 10^{-3}$ & $2 \times 10^{-4}$ & $5 \times 10^{-5}$ & $1 \times 10^{-6}$ \\
\hline$S_{10}$ & $3 \times 10^{-2}$ & $4 \times 10^{-4}$ & $8 \times 10^{-5}$ & $4 \times 10^{-6}$ & $4 \times 10^{-8}$ \\
$G_{10}$ & $1 \times 10^{0}$ & $2 \times 10^{-1}$ & $3 \times 10^{-2}$ & $7 \times 10^{-3}$ & $4 \times 10^{-4}$ \\
\hline$S_{12}$ & $2 \times 10^{-2}$ & $3 \times 10^{-4}$ & $5 \times 10^{-6}$ & $3 \times 10^{-6}$ & $4 \times 10^{-8}$ \\
\hline$G_{20}$ & $1 \times 10^{0}$ & $1 \times 10^{-1}$ & $1 \times 10^{-2}$ & $3 \times 10^{-3}$ & $8 \times 10^{-5}$ \\
\hline$E_{10 a c t}$ & $3.5449 \ldots$ & $-1.5772 \ldots$ & $-0.37231 \ldots$ & $0.59081 \ldots$ & $0.53173 \ldots$ \\
\hline
\end{tabular}

5. Extension to Doubly Infinite Range Integrals. In this section we deal with integrals of the form

$$
J[f]=\int_{-\infty}^{+\infty} w(x) f(x) d x,
$$

where $w(x)$ is an even function of $x$ and all its moments exist. Then

$$
H(z)=\int_{-\infty}^{+\infty} \frac{w(x)}{z-x} d x=2 z \int_{0}^{\infty} \frac{w(x)}{z^{2}-x^{2}} d x \sim \sum_{i=1}^{\infty} \frac{\nu_{i}}{z^{2 i-1}} \quad \text { as } z \rightarrow \infty
$$

where

$$
\nu_{i}=2 \int_{0}^{\infty} w(x) x^{2 i-2} d x, \quad i=1,2, \ldots
$$

If we let $w(x)=|x|^{\beta} e^{-x^{2}}$ and make the change of variable $x^{2}=t$ in (5.2), then

$$
H(z)=z \int_{0}^{\infty} \frac{t^{(\beta-1) / 2} e^{-t}}{z^{2}-t} d t
$$


and

$$
\nu_{i}=\left[\left(\frac{\beta-1}{2}\right)+i-1\right] !, \quad i=1,2, \ldots .
$$

This $H(z)$ is the same as that for $w(x)=x^{\alpha} e^{-x}$ dealt with in Sections 2, 3 and 4, with $\alpha=(\beta-1) / 2$ and $z$ replaced by $z^{2}$. If we let $A_{0}=0, A_{r}=\sum_{i=1}^{r} \nu_{i} / z^{2 i-1}$, $r=1,2, \ldots$, and $R_{r}=\nu_{r} z^{-2 r+1} r^{-1}, r=1,2, \ldots$, then the approximations $T_{k, n}$ to $H(z)$ are again very accurate. Of course, $T_{k, n}$ also in this case is a rational function of $z . T_{k, n}$ has simple poles only when $n=1,2$. When $n=1$, the numerator of $T_{k, n}$ is an odd polynomial of degree $\leqslant 2 k-1$ and its denominator is an even polynomial of degree $2 k$. The zeros of the denominator are $\pm \sqrt{\xi_{i}}, i=1,2, \ldots, k$, where $\xi_{i}$ are the zeros of $((d / d \xi) \xi)^{k} L_{k}^{(s)}(\xi), s=(\beta-1) / 2$. When $n=2$ the numerator of $T_{k, 2}$ is an even polynomial of degree $\leqslant 2 k$ and its denominator is an odd polynomial of degree $2 k+1$. In this case the zeros of the denominator are 0 and $\pm \sqrt{\xi_{i}}, i=1,2, \ldots, k$, where $\xi_{i}$ are the positive zeros of $((d / d \xi) \xi)^{k}\left[L_{k}^{(s)}(\xi)\right]$, $s=(\beta-1) / 2+1$. Therefore, the rational approximations $T_{k, 1}$ and $T_{k, 2}$ give rise to numerical quadrature formulas with $2 k$ and $2 k+1$ abscissas, respectively. For these formulas the weights corresponding to the nonzero abscissas $\pm \sqrt{\xi_{i}}$ are equal. Numerical computations with these formulas show that they are practically as efficient as the corresponding Gaussian formulas.

6. Concluding Remarks. In this work we have used the approach of Sidi [8] to obtain new numerical quadrature formulas for infinite range integrals of the form $\int_{0}^{\infty} w(x) f(x) d x$, where $w(x)=x^{\alpha} e^{-x}, \alpha>-1$, or $w(x)=x^{\alpha} E_{p}(x), \alpha>-1, \alpha+p$ $>0$. We have shown that the abscissas of these formulas are related to the generalized Laguerre polynomials and furthermore we have proved that they are distinct and lie in the interval of integration. The weights of these formulas turn out to be all positive although no proof of this is available yet. We have shown that these formulas are of interpolatory type. An important advantage of these formulas is that for a fixed value of $\alpha$, both $w(x)=x^{\alpha} e^{-x}$ and $w(x)=x^{\alpha} E_{p}(x)$ have the same set of abscissas independent of $p$. Finally, comparison of the new formulas with the Gaussian formulas indicate that the former, on the average, are better than the latter.

Acknowledgement. The computations for this paper were carried out on the IBM-370 computer at the Computation Center of the Technion, Israel Institute of Technology, Haifa, Israel.

Computer Science Department

Technion-Israel Institute of Technology

Haifa, Israel

1. M. Abramowtrz \& I. A. Strgun, Handbook of Mathematical Functions with Formulas, Graphs, and Mathematical Tables, Nat. Bur. Standards, Appl. Math. Series, No. 55, U. S. Government Printing Office, Washington, D. C., 1964.

2. G. BAKER, JR., Essentials of Padé Approximants, Academic Press, New York, 1975.

3. S. Chandrasekhar, The Transfer of Radiant Energy, Clarendon Press, Oxford, 1953.

4. B. DANLOY, "Numerical construction of Gaussian quadrature formulas for $\int_{0}^{1}(-\log x) \cdot x^{\alpha} \cdot f(x)$ $\cdot d x$ and $\int_{0}^{\infty} E_{m}(x) \cdot f(x) \cdot d x$," Math. Comp., v. 27, 1973, pp. 861-869.

5. W. GaUTSCHI, "Algorithm 331, Gaussian quadrature formulas," Comm. ACM, v. 11, 1968, pp. 432-436. 
6. D. LEVIN, "Development of non-linear transformations for improving convergence of sequences," Internat. J. Comput. Math., v. B3, 1973, pp. 371-388.

7. A. SiDI, "Convergence properties of some nonlinear sequence transformations," Math. Comp., v. 33, 1979, pp. 315-326.

8. A. SIDI, "Numerical quadrature and nonlinear sequence transformations; unified rules for efficient computation of integrals with algebraic and logarithmic endpoint singularities," Math. Comp., v. 34, 1980, pp. 851-874.

9. A. SiDI, "Analysis of convergence of the T-transformation for power series," Math. Comp., v. 34, 1980, pp. 833-850.

10. A. SiDI, Converging Factors for Some Asymptotic Moment Series That Arise in Numerical Quadrature, TR \# 165, Computer Science Dept., Technion, Haifa. 